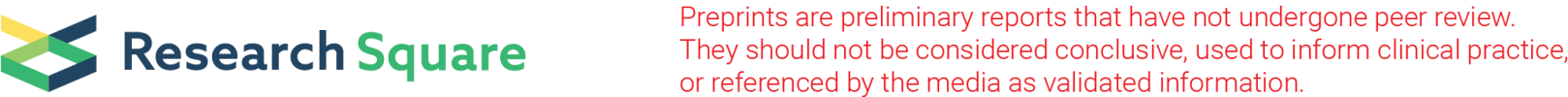

\section{The factors associated with healthy eating behaviors among people with cardiovascular metabolic risk factors: a mixed method study}

Leila Sabzmakan ( $D$ l.sabzmakan@gmail.com )

Alborz University of Medical Sciences https://orcid.org/0000-0003-0642-9548

Mohammad Asghari Jafarabadi

Tabriz University of Medical Sciences

Akbar Nikpajoh

Shahid Rajaee Teacher Training University

Tahereh Kamalikhah

Semnan University of Medical Sciences and Health Services

\section{Research}

Keywords: Healthy eating, Predisposing, Enabling, Reinforcing, Cardiovascular risk factors

Posted Date: December 10th, 2019

DOI: https://doi.org/10.21203/rs.2.18393/v1

License: (c) (1) This work is licensed under a Creative Commons Attribution 4.0 International License. Read Full License 


\section{Abstract}

Background: Healthy eating plays a vital role in the management of metabolic risk factors, such as diabetes, hypertension, and dyslipidemia. The aim of this study was to investigate the factors associated with healthy eating among people with cardiovascular metabolic risk factors.

Method: This study was a mixed method research (qualitative and quantitative). In the qualitative phase, 50 people who had at least a metabolic risk factor and referred to the Diabetes Centers of Karaj, Iran were interviewed based on PRECEDE framework to explain the causes of following/not following healthy eating. In the quantitative phase, first, we developed a questionnaire based on the findings of the qualitative phase, and its validity and reliability was assessed. Then 450 samples completed the questionnaire in order to identify factors related to healthy eating. The qualitative data analysis was done using directed content analysis. In addition, SPSS ver.17 and AMOS ver.18 software were used to analyze the quantitative data.

Results: The results of quantitative part showed only $51.07 \%$ of participants followed healthy eating behaviors. Both the findings of the quantitative and qualitative indicated that predisposing factor was the most important determinant of healthy eating behaviors. There was a significant relationship with large effect size between predisposing factor and healthy eating behaviors $(\beta=1, P=0.001)$. Among predisposing factors, self-efficacy $(\beta=0.49, P=0.001)$ and perceived barriers $(\beta=-0.33, P=0.001)$ were the most significant predictors. However, the findings of quantitative and qualitative phases did not confirm each other for enabling and reinforcing factors. This means in the qualitative phase, enabling factor was perceived as a deep structural determinant of healthy eating, but in the quantitative phase, reinforcing factor $(\beta=0.67, P=0.001)$ was reported as a significant social predictor.

Conclusion: Although personal determinants were the most dominant determinants of healthy eating behaviors, not sufficient to explain eating behaviors. Therefore, social and structural factors have to be considered for promotion of healthy eating behaviors.

\section{Background}

Cardiovascular diseases (CVDs) are the number one cause of death around the world. Per year, a lot of people die from CVDs more than any other cause(1). According to WHO, 17.9 million people died from CVDs in 2016, representing $31 \%$ of all global deaths(1). It is predicted that by 2030 , roughly 23.6 million people will have died from CVD, especially coronary heart disease and stroke(2). It is estimated that $54 \%$ of deaths related to non-communicable diseases in the Eastern Mediterranean Region are due to cardiovascular diseases. The prevalence of cardiovascular diseases is due to sedentary lifestyles and common risk factors, such as hypertension, diabetes and hypercholesterolemia(3). Lipid disorders, high blood pressure, and type 2 diabetes (T2D) have been identified as significant predictors of CVDs(4).

In addition to obesity, diabetes, hypertension, and dyslipidemia, unhealthy dietary patterns (i.e., processed foods, added sugars, excessive use of salt, unhealthy fats, low consumption of fiber, fruit and vegetables) 
develop CVD (5). On the other hand, healthy diet plays a crucial role in the management of metabolic risk factors, such as diabetes, hypertension, and dyslipidemia. At the end of the twentieth century, it was reported that managing of behavioral risk factors could only prevent $40 \%$ to $70 \%$ of all premature deaths, one-third of all cases of acute diabetes and two-thirds of chronic diabetes(6). Moreover, since the beginning of the twenty-first century, behavioral patterns are still the most unique factors affecting health (6). Researchers have repeatedly demonstrated that people find it difficult to follow dietary recommendations(7). Barriers of dietary adherence are in a wide range of psychosocial issues including intra and interpersonal factors, economic constraints, and cultural preferences, social influences, environmental conditions and myriad psychological factors(7). Researchers identified the factors related to healthy eating, but they did not use a theory to understand health behavior change on the basis of psychosocial processes(8-10). Indeed, interventions based on theory specify the presumed influences on mediators of behavior that should to be changed in interventions(11). The PRECEDE framework was developed by Green and colleagues in 1970(12). In this model, the factors associated with behavior are classified as predisposing, reinforcing and enabling(12). The use of PRECEDE model is a logical model which is used to analyze the determinants of a behavior(13). Identifying behavioral determinants will help planners in designing future interventions to select the most appropriate methods and applications to address these determinants and reduce unhealthy behaviors (13). This study investigated the factors associated with healthy eating behaviors among people with cardiovascular metabolic risk factors on the basis of PRECEDE framework.

\section{Methods}

The current study was a mixed method research (qualitative and quantitative). Mixed method researches provide more comprehensive evidence to examine a problem and its behavioral determinants(13). Creswell and colleagues introduced six major mixed method designs (14). In current study, a sequential transformative design with the exploratory sequential features was used since authors applied PRECEDE framework and carried out the qualitative study before than the quantitative study.

The Qualitative phase: A directed content analysis was used to obtain the participants 'perceptions about the causes of following or not following their healthy eating behaviors. The aim of directed content analysis is to validate or extend a conceptual theoretical framework (15). In this approach, an initial coding begins with existing theory and the theory helps to focus on research questions(15). The participants in the qualitative phase were 50 people who had at least one cardiovascular metabolic risk factor for at least one month. They were selected using purposive sampling with maximum variation in terms of (gender, age, education level, occupation and type of disease) among the people who referred to the Diabetes Centers of Karaj-Iran in 2014. If one' blood pressure at two different times was equal to or greater than $14090 \mathrm{mmHg}$; they were diagnosed with hypertension. If the level of one's fasting blood sugar (FBS) was $100 \leq \mathrm{FBS}<126 \mathrm{mg} / \mathrm{dl} 1$; they were diagnosed with pre-diabetic. If their FBS level in two times was equal or higher than $126 \mathrm{mg} / \mathrm{dl}$; they were diagnosed with diabetes. The people with triglyceride level higher than $200 \mathrm{mg} / \mathrm{dl}$, cholesterol equal to or higher than $200 \mathrm{mg} / \mathrm{dl}$, LDL cholesterol higher than $130 \mathrm{mg} / \mathrm{dl}$ and HDL cholesterol equal to or less than $40 \mathrm{mg} / \mathrm{dl}$ were diagnosed with hyperlipidemia.(16). 
The people who were illiterate and suffered from cardiovascular disease and stroke were excluded from the study. The qualitative data was collected via in-depth semi-structured interviews during 6 months. The interview guide was consisted of open-ended questions based on subcategories of predisposing, enabling and reinforcing of PRECEDE framework(17). The interviews continued until data saturation and was recorded, transcribed, reviewed, coded, and immediately analyzed. In order to carry out directed content analysis, the important statements were underlined to identify the initial codes that existed in the interview text. In the next phase, these initial codes were placed into subcategories of PRECEDE model and then into its three main categories including; predisposing factors(18),enabling and reinforcing factors(19). Predisposing factors are antecedents to behavior that provide the motivation for the behavior and include knowledge, attitudes, beliefs, preferences and self-efficacy(12). Reinforcing factors are those which following a behavior that provide continuing reward for the persistence or repetition of the behavior. They include social support, peer influence, significant others, and favorable behavioral outcomes(12). Enabling factors are antecedents to behavioral or environmental change and include programs, services, essential resources and the new skills(12). Placing the codes in subcategories were based on ecological and educational diagnosis phase of PRECEDE model(17). Validity of the data was done by assessing the dependability, reliability, conformability and credibility. All patients who participated in the study were informed about the objectives of research and informed written consent was obtained from all of them. The participants were reassured about confidentiality and anonymity of their information.

The quantitative phase: First, the face validity, content validity, and construct validity of items that were generated from the qualitative phase were assessed. In addition, reliability of the items was assessed by internal consistencyand test-retest. The score of face validity was computed based on impact score of each item and a score equal or greater than 1.5 was considered as reasonable(20) by 10 patients. The content validity index $(\mathrm{CVI})$ and content validity ratio (CVR) of items were also investigated by 10 experts in health education and nutrition. The score of CVI was calculated on the basis of the simplicity/clarification and relevancy of each item and a score equal to or higher than 0.79 indicated an appropriate content validity(20). Moreover, the score of CVR was computed based on the necessity of each item and a CVR score equal or higher than 0.52 was envisaged a good content validity(20). The construct validity of questionnaire was conducted via exploratory factor analysis and confirmatory factor analysis(20). Viramax rotation was used for factor analysis and 3 factors including predisposing, reinforcing and enabling were confirmed for the questionnaire. Cronbach's Alpha coefficient (0.7) indicated a good internal consistency for this questionnaire. Also, Spearman Brown correlation coefficient (0.76) showed good test retest reliability. Second, the standardized questionnaire including three sections; demographic characteristics, clinical outcomes, items of predisposing, enabling and reinforcing factors, and eating behaviors was created (21). We used the questionnaire of Isfahan Cardiovascular Center for measuring eating behaviors, and for scoring the behaviors, world food index (GDI), fat consumption index $(\mathrm{FCl})$ and meat consumption index (MCl) were used(22).

Finally, a cross-sectional study was done in order to assess the relationship between predisposing, enabling and reinforcing factors with healthy eating behaviors. The participants were selected through 
mixed method sampling. It means the Karaj city was divided into three regions: north, central, and south, representing high, medium and low socioeconomic status. Through a list of the Diabetes Centers, two centers were randomly selected from each region. In totally, 6 the Diabetes Centers were selected. Sample size was also calculated based on Walts and colleagues; they have suggested 3-10 subjects for per item of the questionnaire (20). This means in the current study 5 subjects were selected for each item of the questionnaire and totally 450 people completed the questionnaire through interview. All participants were justified about the research method, its confidentiality and goals of the study and informed written consent was obtained from all of them. The data was analyzed using SPSS ver.17 as well as structural equation model was applied using AMOS ver.18.

\section{Results}

Qualitative findings: The average age of participants in this phase was $46.5 \pm 5.97$. All interviews were analyzed and 176 codes were placed into subcategories and three main categories of PRECEDE model.

\section{Predisposing factors:}

Knowledge: Most participants found out that their unhealthy eating habits could result in their health problems such as diabetes, obesity, dyslipidemia and hypertension. They realized the benefits of having a sensible eating plan and its importance in controlling their health problem. Most participants were unaware of healthy and unhealthy foods. The existence of dietitian at the Diabetic Centers helped them to increase their comprehension of healthy foods. "I didn't know how to control my lipid level using diet. Since I attended the Diabetic Center, the dietitian improved my awareness". (Female-43 Aged- metabolic syndrome).

Attitude: Most participants had positive attitude towards unhealthy foods. For example, they would prefer fried and fatty foods to boiled and steamed ones. Also, they had tendency to excessive use of salt and sugar. Some participants especially women preferred to use fat instead of oil for cooking. They believed that oil would stick to coronary arteries and could be harmful to their health. E.g. "I'm always taking fat for cooking because oil sticks to the oven and it is hard to clean it. On the other hand, fat can be cleaned easier when it shed gas. (Female, 47-years-old, pre-diabetes and hyperlipidemia)".

Susceptibility and severity_perceived: The people with diabetes and metabolic syndrome were more worried about complications related to diabetes such as lower limb amputation, blindness, and kidney failure compared with other participants. If diabetics saw these complications in their surroundings, they would perceive more risk and followed healthy eating. "I am mainly worried of my diabetes as my father also had diabetes; he suffered a lot and lost his legs and went blind, and died from it, so I try to adhere the healthy diet forever" (Male - diabetic and hyperlipidemia)

Perceived benefits: Most participants believed that in complying with the healthy eating behaviors controlled their health problem, prevented or reduced medication use, managed their weight, prevented or decreased complications associated with their illness, reduced the cost of health care. "I think that being 
on diet leads to low fat and blood sugar, low blood pressure, losing weight properly, and being fit, when I was overweight, it caused high fat and blood sugar. From when I heard that I'm a diabetic, I do not eat sugar, I eat more vegetables and I eat red meat and fatty foods less, Sometimes I eat barley bread. (Female, 43-years-old, Metabolic Syndrome)".

Perceived barriers: Many participants found out the reasons for poor adherence to healthy eating such as dietary difference between patient and family, diet fatigue, feeling weak when dieting, having a tight schedule, laziness, psychological problems such as depression, stress and anxiety. In addition, most interviewees believed that they were exposure to contradictory nutrition messages that associated with ambiguity about nutrition recommendations. "When I cook for my family, I cannot resist not eating; it is a little hard to cook two different type of food at the same time forever. (Female, 43-years-old, Metabolic Syndrome)".

Self-efficacy: The people with diabetes, metabolic syndrome, hypertension and hyperlipidemia declared more self-efficacy for following healthy eating compared with those who had prediabetes, pre hypertension and low blood cholesterol or triglyceride. Indeed, diabetes patients due to fear of complications related to their illness had more self-efficacy. However, most patients stated low selfefficacy and adherence to healthy eating at social events, parties and during travelling. Some participants believed that they indulged in foods under pressure and when they were alone at home. "Unfortunately, party itself is a problem, you are force to eat. On the one hand, stress makes me eat more, the food which my wife prepares for themselves and put it on the table was just too tempting, when I am at home and have nothing to do, I need to eat and be full. A sometimes when I'm so hungry, I overeat (Male, 49-year-old, metabolic syndrome) ". Moreover, most participants believed that they could not maintain healthy eating on a regular basis. "When my doctor says that my blood sugar and lipid is normal, so I am not going to maintain my regular diet because I think my illness is improved, and I do not any problem, so I can eat everything (Male49-years-old, Metabolic Syndrome) ".

\section{Enabling factors:}

Existence and Access to Resources: The majority of interviewees believed that having access to the dietitian at the Diabetes Center was very helpful to increase their knowledge about healthy foods. In addition, most of them stated the lack of educational materials such as a booklet or brochure about healthy foods. They said that at the Diabetes Centers, the dietitian only gave them a sheet of healthy foods that did not include enough information. Some women or their husbands mentioned to the lack of healthy cooking classes to prepare a healthy food. "The screening program at the Diabetes Center is so good, if this is not, I have no idea that my blood lipid and sugar is high, the free diet consultation which the dietitian gave it to the patients, is so good and give us more information, but if the instructions become like a booklet, I was most interested to read them. (Female, 52 years old, diabetes, and hyperlipidemia)".

New skills: Although many participants benefited from necessary awareness to choose healthy foods, they were unable to read food labels and calculate their daily calorie needs. Moreover, some of them 
claimed that did not have essential skills to plan healthy regimes on a regular basis. Some women or their husbands said that they did not know how to prepare healthy meals, or they indulged in cooking and served plenty of food for the family members. "One problem is that in the house my wife cooks too much food, she inherited it from her mother, and she serves too much food for all of the family members and we will eat all of them, this is a very bad habit. (Male, 37 years-old, hyperlipidemia)".

Rules and Policies: The high cost of healthy foods was one of main contributing factor for not following healthy eating. "If someone wants to follow his diet should have a good financial situation, for example the dietitian told me to eat white meat, but I cannot purchase them regularly, the cost of fish, sesame oil, and canola oil, are too high. (Female, 43 years-old, diabetes)". Furthermore, most respondents believed that there were fatty foods, soft drinks and sweets at parties and celebrations. So, they could not adhere a healthy regime at these events. They also believed that there were overly processed foods and fast foods in society and especially young families had tendency towards them. In addition, some participants felt that places which have sold healthy foods such as low-fat dairy products, diet bread, all kinds of fresh vegetables were not easily accessible and had to commute a long distance for purchasing healthy foods. "Unfortunately, the party itself is a problem, Fried and fatty foods, all kinds of jellies, drinks, were found in these parties, you cannot be on diet and do not eat in these situations (Male, 49-year-old, diabetes)".

Moreover, some interviewees believed that one of main reasons for their health problems was low quality of foods in the markets. They believed that the use of artificial fertilizers, consumption of processed foods and additives, the use of hormones in food production, and fake foods have increased their chances of getting metabolic diseases. Many participants also felt that the authorities did not usually control safety and quality of foods. "Nowadays, all type of foods has chemicals additives, even potato, fruits and wheat. At old times, there was no chemical additives, one of these diseases, such as fat and diabetes were not existing, now when you eat rice you will get diabetes, (Male, 45 years-old Metabolic Syndrome)."

\section{Reinforcing factors:}

Social support: Most participants stated that the dietitians, family members, and peers encouraged them to follow healthy eating. They said when the family members such as wife/husband, children, and parents did not support them to adhere healthy eating, their motivation diminished. The majority of interviewees stated that their family only advised them to follow healthy eating, but they did not accompany the patients. "My children are very closely asking me to be on diet, my husband eats the diet foods but the problem is: my children never eat it, they say that we do not like boiled foods. (Female, 54 years-old, diabetes, and hyperlipidemia)".

Behavioral outcomes: The patients in complying with the dietary regimes experienced positive outcomes such as weight loss, control of blood sugar, blood lipid and blood pressure, feeling of pleasure, and reducing of the symptoms related to their health problems. Therefore, experiencing these positive results stimulated them to follow healthy eating behaviors. "The experience that I earn e.g. I will diet for 
one month then I see their blood sugar or blood lipid become low or I lose weight, my enthusiasm increases" (Man, aged 49, metabolic syndrome).

\section{Quantitative findings:}

The mean age of the participants in this study was $51.43 \pm 7.44$. Among 450 people who participated in the survey, 329 were female $(73.1 \%)$ and 121 were male (26.9\%). The findings of the quantitative phase showed the mean of score for healthy eating behaviors was 6.64 (in the range of $0-13$ ), this means roughly $51.07 \%$ of participants follow healthy eating behaviors. The characteristics of participants 'health problems have been shown in table 1.

In table 2, structural equation modeling has showed that the relationship between predisposing $(\beta=1$., $P=0.001)$ and reinforcing $(\beta=0.67, P=0.001)$ with healthy eating behaviors significantly, and their effects size were respectively large and moderate. In the predisposing categories, the determinants of knowledge $(\beta=0.14, P=0.005)$, attitude $(\beta=0.26, P<0.001)$, perceived severity $(\beta=0.30, P=0.001)$, perceived barriers $(\beta=$ $-0.33, P=0.001)$ and self-efficacy $(\beta=0.49, P=0.001)$ were significant. However, the perceived barriers and self-efficacy were the strongest predictors of healthy eating. In the enabling categories, the laws and policies $(\beta=0.36, P=0.01)$, and new skills $(\beta=0.58, P=0.001)$ were significant determinants. In the reinforcing category, social support $(\beta=0.72, P=0.001)$ and behavioral outcomes $(\beta=0.17, P=0.005)$ were significant determinants, and social support was the strongest predictor. Therefore, these determinants should to be targeted in design of educational interventions in order to promote healthy eating behaviors.

\section{Discussion}

This study aimed to investigate the factors associated with healthy eating behaviors among people with cardiovascular metabolic risk factors based on PRECEDE framework. First, predisposing or personal factors were major contributors of healthy eating behaviors. In this study predisposing category was the most dominant predictors of healthy eating and its effect size was large. Among predisposing factors, knowledge was not a main predictor of adherence to healthy eating. Although there was a significant relationship, its effect size was small. Indeed, most participants knew what foods were beneficial and harmful for their health, but only half of them followed healthy eating behaviors. The studies (23-26) indicated that in spite of having knowledge about healthy foods, the participants did not follow healthy eating behaviors on a regular basis. The participants who had higher awareness and healthy literacy; they perceived the importance of healthy eating in controlling their health problem and were stimulated to follow dietary regimes. Andric and Vuleti (27)supported health literacy as a main factor in complying with health-related behaviors. Therefore, awareness cannot solely guarantee the behavior change will occur. In addition, attitude as a substantial component of behavior is defined in terms of people's overall evaluations of performing behavior(12). While the majority of sample believed that following healthy eating behaviors was good and useful for their health, they had positive attitude towards unhealthy foods. Indeed, they believed that fried foods, sugar, carbohydrate, and fast foods were more delusions than healthy foods. However, most of them declared that their health problem was a main motivation to 
follow healthy eating behaviors. This study indicated attitude as a significant determinant of healthy eating, but its effect size was small. As a result, the participants who had positive attitude toward healthy eating behaviors were more likely to adhere those behaviors (28).

Furthermore, susceptibility refers to the perception of vulnerability to a health problem and its complications, and severity is the perception of health problem as a serious illness. In current study, people with diabetes and metabolic syndrome due to fear of complications related to their illness considered their health problem more serious and followed healthy eating behaviors. This study showed susceptibility was not an important determinant of adherence to healthy eating, whereas perceived severity was a significant predictor. In the study by Adejoh (29) perceived severity had significant relationship with dietary adherence in the patients with diabetes. The study by Becker (30) found out reverse relationship between perceived severity and behavior change. That study reported high perceived severity as a threat and avoiding of following healthy behavior. Additionally, in current study, while almost all participants benefited from following healthy eating behaviors, the perceived benefit was not a significant predictor of healthy eating. The researches (31-33) also confirmed the perceived benefit was not important predictor of self-care among diabetic patients. Consequently, the perceived benefits have often high score, but the score of behavior is relatively poor. It may be due to the inductive effect of the perceived benefits questions.

Although most participants benefited from following healthy eating behaviors, they reported some obstacles for following health eating behaviors. These barriers were confirmed by other studies $(23,24$, 34-37). The study by Ard and colleagues (38) showed that despite knowledge and beliefs as well as low value of perceived barriers to eat healthy foods and high value of perceived benefits, acculturation was important factors in consumption of fruits, vegetables and the amount of fat intake. This is important because culture influences on food preferences. In this study, perceived barrier was a significant predictor of healthy eating behaviors, but its relationship was negative. According to some articles $(33,37,39-42)$ can conclude the fewer barriers, the healthier eating behaviors. Finally, the role of self-efficacy in starting and maintaining healthy behaviors has been demonstrated by several studies $(43,44)$. According to Bandura (12) self-efficacy is defined one's belief in one's ability to succeed in specific situations or accomplish a task. The current study indicated self-efficacy as a significant predictor along with moderate effect size for following healthy eating behaviors. In some articles $(41,45-47)$, self-efficacy was also the strongest predictor of self-care behaviors in diabetes patients. It means individuals who have high self- efficiency to adhere healthy behaviors; they more perform that desired behaviors.

Environmental factors were another main contributor which influenced on healthy eating behaviors. The environmental factors in current study were the enabling and the reinforcing factors. In the qualitative phase, while most participants stated that structural or enabling factor was an important cause of eating behaviors, in the quantitative phase, it was not a significant contributing factor and its effect size was small. However, among enabling factors, the laws/policies related to food and skills associated with healthy eating were significant predictors of healthy eating. A lot of participants reported the lack of skills such as meal planning, reading food labels, ability to calculate daily caloric intake, and preparing healthy 
foods. They also criticized policies and laws related to food such as a lack of access to healthy foods, the high cost of healthy foods, and poor safety food supervision. The high cost of healthy foods were line in with other studies $(24,48,49)$. Furthermore, according to researches $(49-52)$, inappropriate structural environment was one of other barriers of healthy eating. Although having access to resources such as the Diabetes Centers was not a significant predictor of healthy eating behaviors in the quantitative phase, it was perceived as a considerable factor by most participants to adhere healthy eating in the qualitative phase.

In addition to enabling factor, reinforcing or social factor was a significant determinant of healthy eating. Among reinforcing factors, social support by family members, dietitians, and general physicians was a significant and great predictor of healthy eating. In the study by Goetz and colleagues (53), role of social support by nurses and general physicians to change healthy diet among diabetes patients was reported. The study by Story and colleagues (51) reported that the social factors including the interaction with family members, friends, peers, and others in community affected food choices through mechanisms such as; imitation model, social support, and social norms. According to Klomegah (54) if the majority of family members, friends and acquaintances followed healthy diet, dietary adherence would be easy for them. Also, the results of study by Noroozi (55) showed interaction with healthcare providers, family and friends were the only sources of support for diabetics patients. Moreover, behavioral outcome was another significant determinants of healthy eating, though, its effect size was small. Behavioral outcomes are positive or negative results following as a consequence of a behavior and strengthen the motivation for a behavior (17). In current study, most patients by following eating behaviors experienced positive results on physiological indexes such as weight, blood sugar, blood lipid and blood pressure. As a result, behavioral outcome was an internal motivator that encouraged the majority of patients to adhere healthy eating.

Limitations: The findings of the qualitative study provided deep understanding of the factors associated with healthy eating behaviors, a point of view that is not accessible in the quantitative researches. The findings of qualitative study just belong to this population and it lacks the ability of generalization, but this limitation was compensated with quantitative part of study. On the other hand, one of the challenges of mixed method studies is integrating theory into a mixed method research. Another limitation in current study was detection bias because the factors related to healthy eating behaviors and dietary behaviors were measured by the use of the questionnaire. These measures were based on the patient reported outcomes and were more likely to increase cognitive measures bias.

\section{Conclusion}

In the qualitative phase of this current study, most participants believed that predisposing and enabling factors were considerable determinants of healthy eating. However, the findings of the quantitative phase showed that predisposing factors especially self-efficacy and perceived barriers and reinforcing categories particularly social support were the most dominant predictors of healthy eating behaviors. However, new skills as an enabling factor were another significant determinant of healthy eating. All in all, 
this study indicated that personal factors were the most important determinants of following healthy eating behaviors. However, social and structural factors have to be considered in order to design educational interventions for promotion of healthy eating. Identifying of factors related to healthy eating can help planners to explore the most appropriate methods in interventions based on theory in order to modify those factors.

\section{Abbreviation}

CVDs: Cardiovascular diseases; T2D: type 2 diabetes; FBS: fasting blood sugar; CVl: content validity index; CVR: content validity ratio; GDI: world food index; FCl: fat consumption index; $\mathrm{MCl}$ : meat consumption index

\section{Declarations}

\section{Acknowledgment}

The authors would like to thank the Vice Chancellor for Research of Yazd University of Medical Sciences, Iran for the financial support of this study and also the community health centers of Karaj for their cooperation

\section{Funding}

There was not financial aid for doing this research.

\section{Availability of data and materials}

The datasets generated during the qualitative study are not publicly available to protect the participants' anonymity, but the quantitative data are attached as supplementary material. The qualitative data can provide from the corresponding author on reasonable request.

\section{Authors' contributors}

LS, AN and TK were involved in the study design and wrote the proposal. LS interviewed the participants and transcribed the interviews. TK performed the quantitative data gathering. LS and MAJ conducted the qualitative and quantitative data analysis. LS, TK and AN wrote the first draft of the manuscript and edited the final manuscript. All authors approved the final manuscript.

\section{Ethics approval and consent to participate}

Ethical approval was obtained from the Ethics Committee of Shahid Sadoghi University of Medical Sciences (No: P/17/1/57802). Participation in this study was completely anonymous and based on written informed consent. 


\section{Declaration of interest}

The authors report no conflicts of interest. The authors alone are responsible for the content and writing of the article.

\section{Consent for publication}

Not applicable.

\section{Author details}

1.Department of Health Education \& Promotion, Alborz University of Medical Sciences, Karaj, Iran., 2. Road Traffic Injury Research Center, Tabriz University of Medical Sciences, Tabriz, Iran., 3. Rajaei Cardiovascular Medical and Research Center, Iran University of Medical Sciences, Tehran, Iran 4. Department of Public Health, Semnan University of Medical Sciences, Semnan, Iran

\section{References}

1. World Health Organization. Cardiovascular diseases (CVDs): World Health Organization; 2017 [Available from: https://www.who.int

2. World Health Organization. Cardiovascular diseases (CVDs). Fact Sheet No. 317. September 2011 2011 [Available from: http://www.who.int/mediacentre/factsheets/fs317/en/index.html.

3. World Health Organization. Cardiovascular diseases | Health topics - WHO EMRO: World Health Organization; 2017 [Available from: www.emro.who.int

4. Carabello BA. Contemporary Reviews in Cardiovascular Medicine. Circulation. 2005;112:432-7.

5. Casas R, Castro-Barquero S, Estruch R, Sacanella E. Nutrition and cardiovascular health. International journal of molecular sciences. 2018;19(12):3988.

6. McKenzie JF, Neiger BL, Thackeray R. Planning, implementing \& evaluating health promotion programs: A primer: Pearson; 2016.

7. Shumaker S, Ockene J, Riekert K. Promoting Dietary Change . In: S. McCann B, E. Bovbjerg V, editors. The handbook of health behavior change: Springer Publishing Company; 2009.

8. Gillison F, Greaves C, Stathi A, Ramsay R, Bennett P, Taylor G. 'Waste the waist': The developmment of an intervention to promote changes in diet and physical activity for people with high cardiovascular risk. British Journal of Health Psychology. 2011.

9. Artinian NT, Fletcher GF, Mozaffarian D, Kris-Etherton P, Van Horn L, Lichtenstein AH, et al. Interventions to promote physical activity and dietary lifestyle changes for cardiovascular risk factor reduction in adults a scientific statement from the American Heart Association. Circulation. 2010;122(4):406-41.

10. Patel M, Patel IM, Patel YM, Rathi SK. Factors associated with consumption of diabetic diet among type 2 diabetic subjects from Ahmedabad, Western India. Journal of health, population, and nutrition. 
2012;30(4):447.

11. Gourlan M, Bernard P, Bortolon C, Romain A, Lareyre O, Carayol M, et al. Efficacy of theory-based interventions to promote physical activity. A meta-analysis of randomised controlled trials. Health psychology review. 2016;10(1):50-66.

12. Glanz K, Rimer BK, Viswanath K. Health behavior and health education: theory, research, and practice: John Wiley \& Sons; 2008.

13. Bartholomew L, Parcel G, Kok G, Gottlieb. PLANNING HEALTH PROMOTION PROGRAMS:An Intervention Mapping Approach EDITION T, editor. San Francisco: Jossey-Bass; 2011.

14. Creswell JW, Klassen AC, Plano Clark VL, Smith KC. Best practices for mixed methods research in the health sciences. Bethesda, MD: National Institutes of Health. 2011;10.

15. Hsieh H-F, Shannon SE. Three approaches to qualitative content analysis. Qualitative health research. 2005;15(9):1277-88.

16. Delavari AR, Mahdavi Hazaveh AR, Norozi Nejad A, Yarahmadi S. National Program for Prevention and Control of Diabetes. Tehran: Nashr; 2007.

17. Green Iw, kreuter MW. Health Program Planning:AN EDUCATIONAL AND ECOLOGICAL APPROACH. EDITION F, editor. New York: Emily Barrosse; 2005.

18. Sabzmakan L, Morowatisharifabad MA, Mohammadi E, Mazloomy-Mahmoodabad SS, Rabiei K, Naseri $\mathrm{MH}$, et al. Behavioral determinants of cardiovascular diseases risk factors: A qualitative directed content analysis. ARYA atherosclerosis. 2014;10(2):71.

19. Sabzmakan L, Mohammadi E, Morowatisharifabad MA, Afaghi A, Naseri MH, Mirzaei M.

Environmental Determinants of Cardiovascular Diseases Risk Factors: A Qualitative Directed Content Analysis. Iranian Red Crescent medical journal. 2014;16(5).

20. Waltz CF, Strickland OL, Lenz ER. Measurement in nursing and health research: Springer Publishing Company; 2010.

21. Isfahan Cardiovascular Center. Evaluation of knowledge, attitude and performance of society about cardiovascular disease and its related risk factors. National Plan of Isfahan Heart Program. In: Center IC, editor. Isfahan: World Health Organization, Planning and Budget Organization, Health Department of Ministry of Health; 2006.

22. Mohammadifard N, Kelishadi R, Safavi M, Sarrafzadegan N, Sajadi F, Sadri GH, et al. Effect of a community-based intervention on nutritional behaviour in a developing country setting: the Isfahan Healthy Heart Programme. Public health nutrition. 2009;12(09):1422-30.

23. Folta S, Goldberg J, Lichtenstein A, Seguin R. Factors Related Cardiovascular Disease Risk Reduction in Midlife and Older Women: AQualitative Study. Prev Chronic Dis. 2008;5(1):1-9.

24. Nakkash R, Soweid RAA, Nehlawi MT, Shediac-Rizkallah MC, Hajjar TA, Khogali M. The development of a feasible community-specific cardiovascular disease prevention program: Triangulation of methods and sources. Health Education \& Behavior. 2003;30(6):723-39. 
25. Green A, Bazata D, Fox K, Grandy S. Health-related behaviours of people with diabetes and those with cardiometabolic risk factors: results from SHIELD. International journal of clinical practice. 2007;61(11):1791-7.

26. Roberts K, Marvin K. Knowledge and attitudes towards healthy eating and physical activity: what the data tell us. National Obesity Observatory, Oxford. 2011.

27. Andric A, Vuleti V. Community Nurse Assessment of Cardiovascular Behavioural Risk Factors - A Qualitative Analysis within the CroHort Study. Coll Antropol. 2012;36:27-34.

28. Naughton P, McCarthy S, McCarthy M. Healthy eating attitudes and healthy living: An examination of the relationship between attitudes, food choices and lifestyle behaviours in a representative sample of Irish adults. Proceedings of the Nutrition Society. 2013;72(OCE4).

29. Adejoh SO. Diabetes knowledge, health belief, and diabetes management among the Igala, Nigeria. Sage Open. 2014;4(2):2158244014539966.

30. Becker MH, Kaback MM, Rosenstock IM, Ruth MV. Some influences on public participation in a genetic screening program. Journal of community health. 1975;1(1):3-14.

31. Gillibrand R, Stevenson J. The extended health belief model applied to the experience of diabetes in young people. British journal of health psychology. 2006;11(1):155-69.

32. Patino AM, Sanchez J, Eidson M, Delamater AM. Health beliefs and regimen adherence in minority adolescents with type 1 diabetes. Journal of pediatric psychology. 2005;30(6):503-12.

33. Dehghani-Tafti A, Mahmoodabad SSM, Morowatisharifabad MA, Ardakani MA, Rezaeipandari H, Lotfi MH. Determinants of self-care in diabetic patients based on health belief model. Global journal of health science. 2015;7(5):33.

34. Marcy T, Britton M, Harrison D. Identification of Barriers to Appropriate Dietary Behavior in LowIncome Patients with Type 2 Diabetes Mellitus. diabetes Ther. 2011;2(1):9-19.

35. López-Azpiazu I, Martines-Gonzalez M, Kearney J, Gibney M, Martinez JA. Perceived barriers of, and benefits to, healthy eating reported by a Spanish national sample. Public health nutrition. 1999;2:209-16.

36. Pawlak R, Colby S. Benefits, barriers, self-efficacy and knowledge regarding healthy foods; perception of African Americans living in eastern North Carolina. Nutrition research and practice. 2009;3(1):5663.

37. Cheng L, Leung DY-P, Sit JW-h, Li X-m, Wu Y-n, Yang M-y, et al. Factors associated with diet barriers in patients with poorly controlled type 2 diabetes. Patient preference and adherence. 2016;10:37.

38. Ard JD, Skinner CS, Chen C, Aickin M, Svetkey LP. Informing cancer prevention strategies for African Americans: The relationship of African American acculturation to fruit, vegetable, and fat intake. Journal of behavioral medicine. 2005;28(3):239-47.

39. Koch $\mathrm{J}$. The role of exercise in the African-american woman with type 2 diabet mellitus: Aplication of the Health Belief Model. J Am Acad Nurse Pract. 2002;14(3):126-30. 
40. Daniel M, Messer LC. Perceptions of disease severity and barriers to self-care predict glycemic control in Aboriginal persons with type 2 diabetes mellitus. Chronic Diseases and Injuries in Canada. 2002;23(4):130.

41. Aljasem LI, Peyrot M, Wissow L, Rubin RR. The impact of barriers and self-efficacy on self-care behaviors in type 2 diabetes. The Diabetes Educator. 2001;27(3):393-404.

42. Morowatisharifabad M, Rouhani Tonekaboni N. The relationship between perceived benefits/barriers of self-care behaviors and self management in diabetic patients. Journal of hayat. 2007;13(1):17-27.

43. Shortridge-Baggett LM. Self-efficacy: measurement and intervention in nursing. Scholarly inquiry for nursing practice. 2001;15(3):183.

44. Krichbaum K, Aarestad V, Buethe M. Exploring the Connection Between Self-Efficacy and Effective Diabetes Self-f Management. The Diabetes Educator. 2003;29(4):653-62.

45. Sarkar U, Fisher L, Schillinger D. Is self-efficacy associated with diabetes self-management across race/ethnicity and health literacy? Diabetes care. 2006;29(4):823-9.

46. Mohebi S, Azadbakht L, Feizi A, Sharifirad G, Kargar M. Review the key role of self-efficacy in diabetes care. Journal of education and health promotion. 2013;2.

47. Jiang $X$, Wang J, Lu Y, Jiang H, Li M. Self-efficacy-focused education in persons with diabetes: a systematic review and meta-analysis. Psychology research and behavior management. 2019;12:67.

48. Chow CK, Lock K, Teo K, Subramanian S, McKee M, Yusuf S. Environmental and societal influences acting on cardiovascular risk factors and disease at a population level: a review. International journal of epidemiology. 2009;38(6):1580-94.

49. Popkin BM, Duffey K, Gordon-Larsen P. Environmental influences on food choice, physical activity and energy balance. Physiology \& behavior. 2005;86(5):603-13.

50. Raine KD. Determinants of healthy eating in Canada: an overview and synthesis. Canadian Journal of Public Health/Revue Canadienne De Sante'e Publique. 2005:S8-S14.

51. Story M, Kaphingst KM, Robinson-O'Brien R, Glanz K. Creating healthy food and eating environments: policy and environmental approaches. Annu Rev Public Health. 2008;29:253-72.

52. Robinson T. Applying the socio-ecological model to improving fruit and vegetable intake among lowincome African Americans. Journal of community health. 2008;33(6):395-406.

53. Goetz K, Szecsenyi J, Campbell S, Rosemann T, Rueter G, Raum E, et al. The importance of social support for people with type 2 diabetes-a qualitative study with general practitioners, practice nurses and patients. GMS Psycho-Social-Medicine. 2012;9.

54. Klomegah RY. The social side of diabetes: The influence of social support on the dietary regimen of people with diabetes. Sociation Today. 2006;4(2):534-53.

55. Noroozi A, Tahmasebi R, Rekabpour SJ. Effective social support resources in self-management of diabetic patients in Bushehr (2011-12). ISMJ. 2013;16(3):250-9.

\section{Tables}


Table1. Characteristics of the participants' health problems

\begin{tabular}{ccc}
\hline Variable & Level & Percentage \\
\cline { 1 - 1 } Cholesterol & & \\
Desirable & 170 & 37.9 \\
High & 278 & 62.1 \\
Triglycerides & & \\
Desirable & 122 & 27.3 \\
Border & 104 & 23.3 \\
High & 221 & 49.4 \\
LDL & & \\
Desirable & 249 & 63.2 \\
Border & 96 & 24.4 \\
High & 49 & 12.4 \\
HDL & & \\
Low & 96 & 21 \\
Good & 248 & 55.4 \\
Desirable & 106 & 23.7 \\
FBS & & \\
Normal & 28 & 6.2 \\
Prediabetes & 125 & 27.9 \\
Diabetes & 295 & 65.8 \\
Blood Pressure & & \\
Normal & 163 & 36.5 \\
Pre-hypertension & 206 & 46.1 \\
High Hypertension & 78 & 17.4 \\
\hline
\end{tabular}

Table.2. Path coefficients of PRECEDE Model construes with healthy eating behaviors

\begin{tabular}{ccccc}
\hline Independent variable & Dependent variable & Standardized coefficients & P-Value \\
\hline Predisposing & Healthy eating & 1 & 0.001 \\
Enabling & Healthy eating & 0.38 & 0.11 \\
Reinforcing & Healthy eating & 0.67 & 0.001 \\
Perceived susceptibility & Predisposing & 0.064 & 0.19 \\
Perceived severity & Predisposing & 0.30 & 0.001 \\
Knowledge & Predisposing & 0.14 & 0.005 \\
Attitude & Predisposing & 0.26 & 0.001 \\
Benefits & Predisposing & 0.051 & 0.19 \\
Barriers & Predisposing & -0.33 & 0.001 \\
Self-efficacy & Predisposing & 0.49 & 0.001 \\
Skills & Enabling & 0.58 & 0.001 \\
Resources & Enabling & 0.012 & 0.85 \\
Laws/Policy & Enabling & 0.36 & 0.01 \\
Social support & Reinforcing & 0.72 & 0.001 \\
Behavioral Consequences & Reinforcing & 0.17 & 0.005 \\
\hline
\end{tabular}


Page 17/17 